
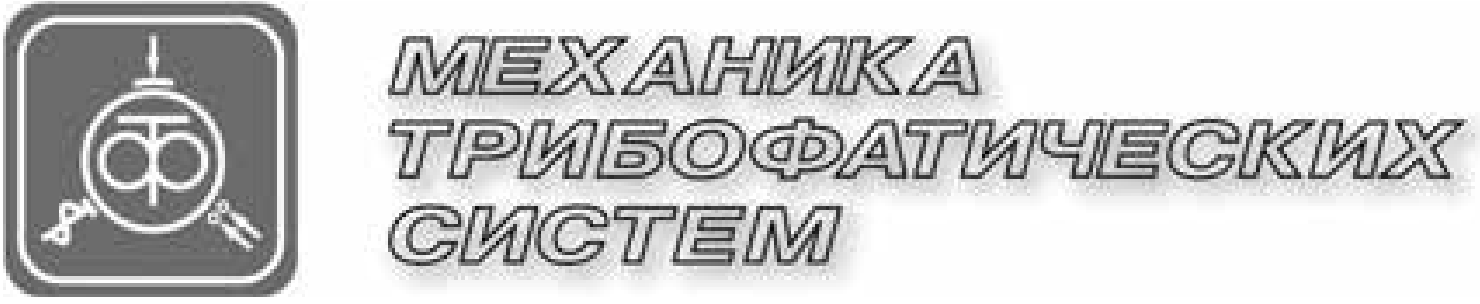

\title{
УДК 536
}

Л.А. СОСНОВСКИЙ, д-р техн. наук, проф.

профессор кафедры «Локомотивы» ${ }^{1}$

E-mail: tribo-fatigue@mail.ru

С.С. ЩЕРБАКОВ, д-р физ.-мат. наук, проф.

профессор кафедры теоретической и прикладной механики ${ }^{2}$

E-mail: sherbakovss@mail.ru

${ }^{1}$ Белорусский государственный университет транспорта, г. Гомель, Республика Беларусь

${ }^{2}$ Белорусский государственный университет, г. Минск, Республика Беларусь

Поступила в редакциию 25.08.2020.

\section{РАЗВИТИЕ ПОНЯТИЯ ОБ ЭНТРОПИИ: ОТ ТЕРМОДИНАМИКИ ДО КОСМОЛОГИИ. СООБЩЕНИЕ 2*. ГИПОТЕЗА ОБ ЭНТРОПИИ В КОСМОЛОГИИ И ЕЕ РАЗВИТИЕ}

Проблемь важнее решения.
Решения могут устареть, а проблемы остаются.

Нильс Бор

B [7] систематизировань классические представления о термодинамической энтропии и изложень современные подходы к оченке трибофатической и механотермодинамической энтропии неаддитивных систем. В данной статье изложена и анализируется концепция об аналогии термодинамики и механики черных дыр, которая позволила дать оценку их (термодинамической) энтропии. Недостаточность этой концепции состоит в том, что термодинамическая энтропия есть характеристика рассеяния энергии, тогда как для черных дыр характерно поглощение энергии и вещества. В этой связи предложено рассматривать горизонт событий как термодинамическую среду, а черную дыру - как трибофатический объект. И тогда система «черная дыра - горизонт событий» представляется как объединенная механотермодинамическая неаддитивная система. Изложень методы оценки полной (механотермодинамической) энтропии и ее составляющих - трибофатической и термодинамической энтропии в механике черных дыр. Применительно к отдельным (конкретным) зонам и объектам Вселенной известная теория Зельдовича соответственно модифицируется: Вселенная - это термодинамическая среда с дискретно распределенными (рассеянными) в ней плотными и/или твердыми телами (объектами) - звездами, галактиками и др. Описаны некоторые примеры поведения такой системы (прямой и обратный эффекты во Вселенной). Особенность действия среды на звезды и, наоборот, действия скопления звезд на среду, т. е. взаимодействие между ними, состоит в том, что оно неньютоновское: действие не равно противодействию. Именно неравенство действия противодействию, которые имеют кардинально различающиеся механизмы и последствия (результать), или, иначе, неуравновешенность Вселенной и определяют в известной мере ее всеобщее движение в пространстве-времени. Изменяющаяся совокупность всех состояний и есть эволючия Вселенной. Анализ возможных стратегий эволюции механотермодинамических систем выполнен на базе фундаментального принципа: повреждаемость всего сущего не имеет мыслимых грании. Этот приничи сформулирован в механотермодинамике и использован в философии для создания обобщенной теории эволющчи материального мира.

\footnotetext{
*Написано по материалам доклада «Развитие понятия об энтропии: от термодинамики до космологии», представленного на пленарном заседании VIII Белорусского конгресса по теоретической и прикладной механике «Механика - 2019» (2. Минск, Объединенный институт машиностроения НАН Беларуси, 18-20 сентября 2019 г.)
} 
Ключевые слова: черная дыра; горизонт событий; термодинамика; механика черных дыр; энтропия термодинамическая, трибофатическая, механотермодинамическая; кониепиия Бекенштейна-Хокинга; планковские длина, площадь, объем; система аддитивная, неаддитивная; теория Зельдовича; эволючия системы по поврежденности; L-риск; $S_{\rho}$-безопасность; Вселенная

DOI: https://doi.org/10.46864/1995-0470-2020-1-54-80-88

Введение. Дальнейшее развитие второго закона термодинамики дано в одной из космологических конщепщий [1-4]. Бекенштейн выдвинул предположение, которое казалось невероятным: в черных дырах может содержаться огромная энтропия $[1,2]$. Цель данной работы состоит в том, чтобы проанализировать эту концепцию и дать ее развитие.

Черные дыры образуются в результате коллапса очень массивных звезд. Последние могут иметь соседей, которые вращаются вокруг коллапсирующей звезды. Потоки газа, испускаемые ими, могут $n a-$ дать на черную дыру, образуя вокруг нее аккреционный диск, который, в свою очередь, нагревается до гигантских температур и генерирует мощное рентгеновское излучение. Такой диск называют горизонтом событий (рисунок $1 a, b$ ). Спутники обнаружили множество источников подобного излучения. Его анализ позволяет понять некоторые свойства черных дыр.

Имеются также доказательства существования сверхмассивных черных дыр в изентрах галактик - их массы в миллионы раз превышают массу Солнца. На ранних стадиях образования галактик эти гигантские черные дыры поглощают все вещество вокруг себя, затягивая его мощнейшими вихрями, которые наблюдаются как квазары. Согласно общей теории относительности - это не просто плотно упакованный объем исходного материала. Это, по-видимому, чистое гравитационное поле. После завершения формирования черной дыры у нее остаются три характеристики, поддающиеся измерению: ее полная масса, скорость вращения и электрический заряд.

Концепция Бекенштейна-Хокинга и ее анализ. Установлена [1-4] аналогия термодинамики и механики черных дыр: энергия, температура и энтропия в термодинамике - характеристики, аналогичные массе, поверхностной гравитащии и площади горизонта событий для черных дыр (рисунок 2). В результате Бекенштейн [1] предложил обобщенное второе начало термодинамики, представляющее собой, по сути, обычное второе начало термодинамики, но с добавлением энтропии черных дыр. Согласно расчетам Хокинга $[3,4]$, энтропия черной дыры равна одной четверти площади ее горизонта, измеренного в единицах планковской площади:

$$
S_{B H}=\frac{1}{4} \frac{A}{L_{p}^{2}} .
$$

А площадь горизонта событий пропорциональна квадрату массы $M$ черной дыры:

$$
A=16 \pi G^{2} M^{2} / c^{4},
$$

где $G$ - гравитационная постоянная; $c$ - скорость света. Нижний индекс $B H$ можно читать как Black Hole (черная дыра), или Bekenstein-Hawking (Бекенштейн-Хокинг).

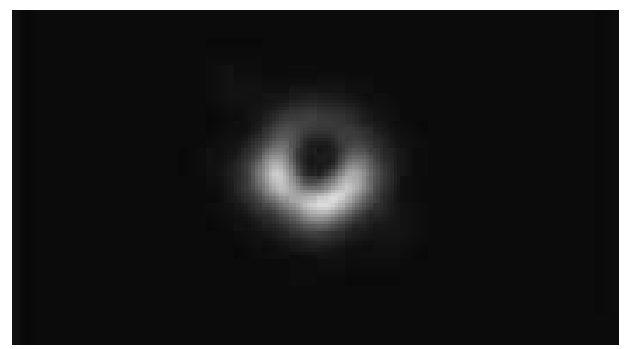

$a$

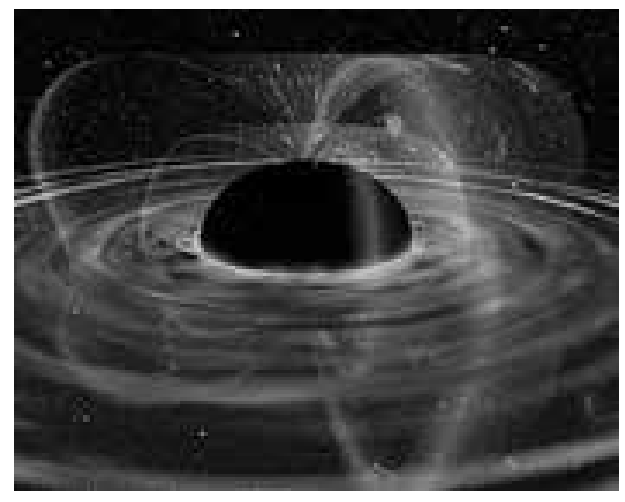

$b$

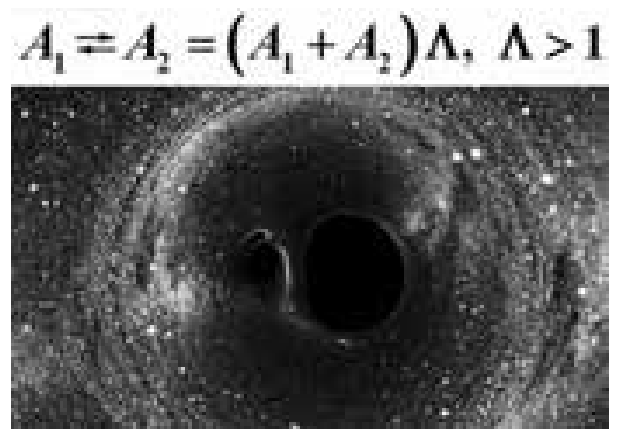

C

Рисунок 1 - Изображения площади горизонта событий и черных дыр: $a$ - первое изображение горизонта событий черной дыры; $b$ - рисунок художника: аккреционный диск горячей плазмы, вращающийся вокруг черной дыры; c- изображение: столкновение черных дыр

Figure 1 - Images of the area of event horizon and black holes: $a-$ the first image of the event horizon of a black hole; $b-$ artist's drawing: an accretion disk of hot plasma rotating around the black hole; $c$ - image: a collision of black holes [a, $c$ - https://42.tut. by/651698?c; $b$ - https://ru.wikipedia.org/wiki/Чёрная_дыра] 
В (1) площадь $A$ горизонта событий выражается в единицах планковской площади $L_{p}^{2}$. Планковская длина $L_{p}$, равная $1,6 \cdot 10^{-35} \mathrm{M}$, есть расстояние, на котором квантовая гравитация начинает играть заметную роль. Планковская площзадь — это квадрат планковской длины. Для черной дыры, масса которой сравнима с массой Солнца, площадь горизонта событий составляет около $10^{77}$ планковских площадей. Это больше известной оценки энтропии всех звезд, газа и пыли во всей галактике Млечный Путь.

Согласно Хокингу, хотя и можно уменьшить массу/энергию вращающейся черной дыры, существует величина, которая всегда либо увеличивается, либо остается неизменной: площадь горизонта событий, которая, по сути, соотносится с размером черной дыры. Это похоже на то, как ведет себя энтропия согласно второму началу термодинамики. Площадь горизонта зависит от сочетания массы, углового момента и заряда. И всякая конкретная их комбинация никогда не уменьшается. Например, если есть две черные дыры $A_{1}$ и $A_{2}$ (см. рисунок $1 c$ ), они могут столкнуться друг с другом и слиться в одну черную дыру $A_{12}$. Однако площзадь нового горизонта событий всегда больше, чем суммарная площуадь двух исходных горизонтов:

$$
A_{12}>\left(A_{1}+A_{2}\right) \text {. }
$$

Но одна большая черная дыра никогда не может распасться на две меньшие, так как в этом случае ее площадь пришлось бы уменышить.

На рисунке 2 мы в принципе указали недостаточность аналогии Бекенштейна для системь черHblx дыр. Рассмотрим этот вопрос более подробно.

Исходя из термодинамических соображений $[5,6]$, формула (1) в механике черных дыр аддитив- на. Однако убедительно показано, что слияние двух черных дыр - процесс заведомо неаддитивный:

$$
F_{\Lambda}\left(A_{1} \rightleftarrows A_{2}\right)=\left(A_{1}+A_{2}\right) \Lambda_{112}=A_{12}, \Lambda_{112}>1,0,
$$

так что, как гласят (3) и (4), $A_{12}>>\left(A_{1}+A_{2}\right)$. Но согласно термодинамике должно быть $A_{12}=\sum_{i=1}^{2} A_{i}$. Что-

бы снять это противоречие, можно принять, что формула (1) относится не к черной дыре как таковой, а только к ее площади горизонта событий. Тогда следует уточнить, что в действительности происходит на горизонте событий: рассеяние энергии или ее поглощение? Ответ прост: преимущественно рассеяние, например, путем мощного рентгеновского излучения (см. выше). В этой связи следует правильно понять и формулу (1). В статистической механике, согласно Больиману (см. анализ в [7]), энтропия пропорциональна логарифму числа независимых и равновероятных микросостояний (конфигураций) $W$ среды с точностью до постоянной. Постоянная $k$ носит его имя: $k=k_{B}$. Именно она обеспечивает одинаковую размерность термодинамической энтропии $S_{T D}$ и правой части уравнения Больимана $S_{T D}=k_{B} \log W$. Формула (1) не предусматривает логарифмирования числа равновероятных конфигураций, содержащихся в среде, как это должно быть по Больциману. Однако в ее правой части просто обнаруживается огромное (безразмерное) число $A_{L}$ специфических конфигураций — планковских площадей, содержащихся в определенной площади горизонта событий $A$ :

$$
A_{L}=\frac{A}{L_{p}^{2}}(\uparrow) .
$$

Нетрудно понять, что число $A_{L}$ подчиняется статистике Больцмана, т. е. $A_{L} \equiv W$. Тогда с точностью

Аналогия Бекенштейна: обобщенное второе начало термодинамики Термодинамическая Черная система дыра

Энергия
(рассеянная)
Температура
$\begin{gathered}\text { Термодинамическая } \\ \text { энтропия }\end{gathered}$ $\begin{gathered}\text { Поверхностная } \\ \text { гравитация }\end{gathered}$

Нелостаточность аналогии Бекенштеінна для системы черных дыр

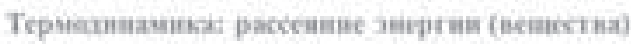

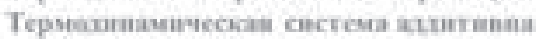

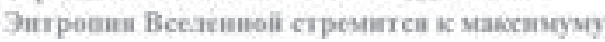


до постоянной формулу (1) следует записать и прочитать так: термодинамическая энтропия $S_{T D}$ пропорииональна логарифму числа планковских площзадей в данной среде:

$$
S_{T D}=k_{A} \log A_{L}(\uparrow) .
$$

При этом, возможно, $k_{A}=k_{B}$. Поскольку формула (6) относится к вполне определенному объекту среде, называемой горизонтом событий, в ней можно конкретизировать обозначение энтропии:

$$
S_{\text {гс }}=k_{A} \log A_{L} .
$$

Развитие гипотезы. Будем искать аналогию между механикой собственно черных дыр (как отдельных объектов) и энтропией. Тогда надо учесть, что черная дыра - это объем очень плотной материи, образование которой обусловлено исключительно поглощением энергии и вещества. Следовательно, энтропию системы черных дыр надо связать с трибофатической энтропией (см. формулу (21) в [7]), которая и является мерой поглощения энергии [8-10]. Поскольку трибофатическая энтропия определяется числом $\omega_{\Sigma}\left(U_{\Sigma}^{e f f}\right) \rightarrow \infty$ необратимых повреждений, обусловленных нагрузками разной природы, то для механики черных дыр ее можно записать аналогично (6):

$$
S_{T F}=k_{s s} \log \omega_{V}=S_{b h}(\uparrow),
$$

где $\omega_{V} \approx \gamma_{1}^{(\omega)} \omega_{\Sigma} d V_{P \gamma}$, что вполне аналогично также уравнениям (1), (6) и (11) в [7]. Здесь $k_{s s}$ подлежит определению (и может быть показано, что $k_{\mathrm{ss}} \neq k_{\mathrm{B}}$ ). Формула (7) гласит: энтропия системы черных дыр $S_{T F}$ пропорииональна (с точностью до постоянной) логарифму необратилых «повреждений в ее объеме» $\omega_{V}$. А численное значение (величину) $\omega_{V}$, применительно к черной дыре, следует определить аналогично (5), а именно

$$
\omega_{V}=\frac{V_{B H}}{L_{p}^{3}}=\omega_{b h} \uparrow .
$$

Таким образом, $\omega_{V}-$ это (безразмерное) число планковских объемов $L_{p}^{3}$, содержащихся в объеме черной дыры $V_{B H}$. Соотношение (8) позволяет отказаться от пока неясного понятия «повреждение черной дыры» путем введения новой конфигуращии (8) числа микросостояний в исследуемом объекте (подобно как в статистической механике по Больиману). Объем черной дыры в виде сферы вычисляется по радиусу Шваријшильда:

$$
R_{S}=2 G_{M} / c^{2},
$$

где $M$ - масса черной дыры; $c$ - скорость света; $G$ - гравитационная постоянная. Тем самым принимается предположение, что комплекс \{система взаимодействуюших черных дыр $\leftrightarrows$ горизонт событий есть неаддитивная механотермодинамическая мультисистема.

Итак, в действительности в механике черных дыр, похоже, следует различать две компоненть энтропии. Одна компонента $\left(S_{T D}=S_{\text {гс }}\right)-$ это энтропия, определяемая рассеянной в площади горизонта событий энергией. А другая компонента $\left(S_{T F}=S_{b h}\right)$ - это энтропия, определяемая поглощенной энергией в объеме черной дыры. Будем считать, что в общем случае в механике черных дыр можно определять обобщенную (неаддитивно объединенную) механотермодинамическую энтропию [7]. Тогда, согласно второму принципу механотермодинамики $[9,10]$

$$
F_{\Lambda}\left(S_{\mathrm{rc}} \rightleftarrows S_{b h}\right)=\Lambda_{T D \backslash T F}\left(S_{\mathrm{rc}}+S_{b h}\right)=S_{M T D} \uparrow,
$$

или, с учетом (6) и (7)

$$
S_{\text {MTD }}=\left(k_{A} \log A_{L}+k_{s s} \log \omega_{b h}\right) \Lambda_{M T D} \uparrow .
$$

Имея (7) и (10a), можно уточнить (понять), смысл параметра $\omega_{V}$ в (8). Согласно (7), статистический подход Больимана оказывается применимым к черной дыре как среде. А согласно (10a), сумма двух слагаемых в скобках должна быть суммой рассеянной (первое слагаемое) и поглощенной (второе слагаемое) частями полной энергии механотермодинамической системь «горизонт событий $\leftrightarrows$ черная дыра». И специфическая особенность этой системы состоит в том, что рассеянная и поглощенная части энергии здесь преимущественно разделены механизмами их образования и способами их существования. Из изложенного следует, что поглощенная черной дырой энергия - это часть рассеянной горизонтом событий энергии; другая ее часть рассеивается в окружающее пространство в виде мощного рентгеновского излучения. Вывод таков: объем собственно черной дыры и есть результат поглощения энергии и вещества, что и отражено в (8) в безразмерном виде. Итак, «повреждение черной дыры» - это просто ее относительный объем, исчисленный в планковских объемах. И поэтому в формулах (10) и (10a) должно быть $\Lambda_{\text {мTD }}>1$. Это соответствует известной энтропийной закономерности [7]: энтропия способна увеличиваться всегда.

Таким образом, можно предложить: когда кратко говорят «механика черных дыр», то подразумевают механотермодинамические системы «черная дыра - горизонт событий».

Исходя из изложенного, следует модифицировать (уточнить) общепринятую ныне космологическую гипотезу Зельдовича $[11,12]$, согласно которой Вселенная представляет собой сплошную и однородную среду. Наша гипотеза: Вселенная - это гигантская механотермодинамическая мультисистема, или, иначе, термодинамическая среда с дискретно распределенными (рассеянными) в ней плотными и/или твердыли телами (объектами) - звездами, галактиками и др., которые взаимодействуют как межну собой, так и со средой. В самом деле, нельзя отрицать, что, например, движение, деградация и распад (по любым причинам) звезд не возмущают межзвездную пространственно-пылегазовую 
среду. Механизмы и последствия такого процесса в значительной мере определяют эволюиию Вселенной. С другой стороны, можно предположить, что состав, строение и состояние пространства, в свою очередь, оказывают влияние (определенное действие) на процессы существования и разложения звезд. Это означает, что во Вселенной имеют место как прямой, так и обратный эффекты [13-17]. Таким образом, действие и противодействие во Вселенной асимметричны. Следовательно, это диалектические $\Lambda$-взаимодействия $[13,18]$.

На примере теории Зельдовича можно еще раз подтвердить большое значение результатов наших «земных исследований» для использования их при построении космологических теорий. Так, напомним: в механике материалов главный объект исследований - материал - считается сплошной однородной и изотропной средой (ср. с теорией Зельдовича). Однако для получения практически полезных результатов (например, для расчета определенных изделий на прочность) гипотеза сплошности заменяется учетом фактического состояния конкретного металла с точно определенными параметрами механических свойств, в том числе характеризующих его прочность в различных направлениях пространства (объема). В космологических исследованиях аналогично изучаются конкретное строение и определенные свойства объектов и систем в изучаемых зонах Вселенной (черные дыры, звездные системы и т. д.).

Изменяющиеся при движении состояния элементов механотермодинамической мультисистемы можно охарактеризовать в классических терминах: сила, энергия, поле, энтропия, повреждение (температура, масса, скорость и др.). Конечно же наши пути познания Вселенной это ее «расчленение на части». От того, как мы сумеем собрать разъятые части в единое целое, зависит соответствие наших знаний истине (в работе [19] введен даже показатель истинности знания).
Таким образом, эволюция систем может быть тождественно описана с использованием таких мощных интегральных характеристик состояния, как энергия, энтропия, повреждение. Но важно, чтобы соответствующие модели были адекватными известным закономерностям развития материального мира - пусть самым простым. Ситуация такова. Всякие необратимые повреждения любой системы реальны. Их можно ощутить, увидеть, измерить и т. д., следовательно, достоверно описать. А материальные носители энергии или энтропии до сих пор неизвестны или даже не существуют [20], их нельзя наблюдать, но их можно вообразить. Именно поэтому считается [21-23], что анализ эволюции систем по поврежденности представляется практически более перспективньлм.

Одна из перспектив: согласно третьему принципу механотермодинамики $[9,10]$, масштаб интегральной поврежденности $V_{i j}$ системы и ее элементов в полной мере характеризует $L$-риск $\rho$ и, значит, безопасность $S_{\rho}$ их существования (жизни). В таблице эти связи охарактеризованы (описаны) количественно [22].

Обобщенная модель эволюционного процесса имеет вид:

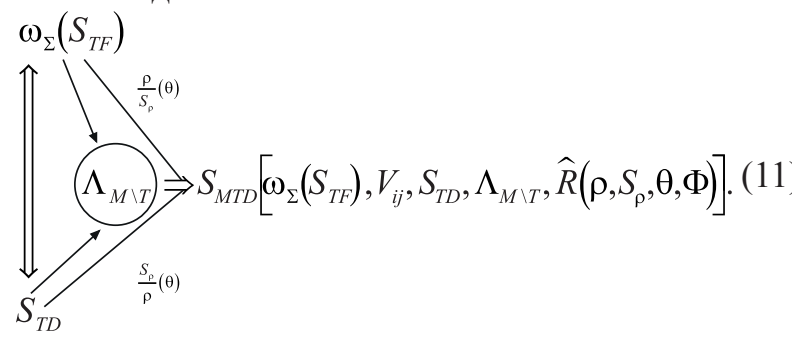

Согласно (11), прогнозируются следующие две стратегии [24] эволюции: $Y$ - стремление к нулевомy $L$-риску $\rho(t) \rightarrow \rho_{0} \rightarrow 0$ признается естественным для Природы и $X$ - стремление к абсолютной безопасности $S_{\rho}(t) \rightarrow S_{d} \rightarrow 1$ признается как всеобщее для любых ее систем. При этом эволюционный процесс определяется не абсолютными значениями $L$-риска и $S_{\rho}$-безопасности, а их

Таблица - Эволюция объектов по повреждаемости: количественный анализ Table — Evolution of objects by damage: a quantitative analysis

\begin{tabular}{|c|c|c|c|c|c|}
\hline \multicolumn{2}{|c|}{ Эволюция системы } & \multirow{2}{*}{$\begin{array}{c}\text { Состояния } \\
\text { поврежденности } \\
\text { Неповрежденное } \\
\omega_{\Sigma}=0=\omega_{0} \\
\end{array}$} & \multirow{6}{*}{$\begin{array}{c}A \text {-эволюция: } \\
\text { развитие необратимых состояний } \\
\text { системы по поврежденности } \\
\text { и ситуаций по безопасности: } \\
\vec{\omega}_{\Sigma}=\vec{\omega}_{\Sigma}\left(U_{\Sigma}^{e f f}\right) \stackrel{t}{\Rightarrow}_{h} \in \infty \\
d_{\omega}^{*}=e^{-K \omega_{\Sigma}^{*}} \Rightarrow \frac{1}{h} \in 0\end{array}$} & \multirow{2}{*}{\multicolumn{2}{|c|}{$\begin{array}{c}\text { Ситуации по безопасности } \\
\text { Абсолютная безопасность } \\
S_{\rho}=1=S_{d}\end{array}$}} \\
\hline \multirow{3}{*}{ 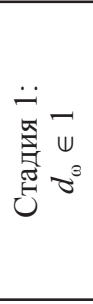 } & A-состояние & & & & \\
\hline & B-состояние & $\begin{array}{c}\text { Поврежденное } \\
0<\omega_{\Sigma}(t)<1\end{array}$ & & \multirow{4}{*}{ 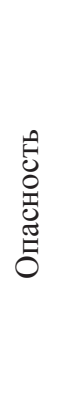 } & $\begin{array}{c}\text { Ограниченная } \\
\text { безопасность } \\
1>S_{\rho}>0 \\
\end{array}$ \\
\hline & $C$-состояние & $\begin{array}{l}\text { Предельное } \\
\omega_{\Sigma}=1=\omega_{\lim }\end{array}$ & & & $\begin{array}{c}\text { Нулевая безопасность } \\
S_{\rho}=0=S_{0}\end{array}$ \\
\hline \multirow{2}{*}{ 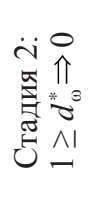 } & $D$-состояние & $\begin{array}{l}\text { Запредельное } \\
1<\omega_{\Sigma}^{*}(t)<\infty\end{array}$ & & & $\begin{array}{c}\text { Отрицательная } \\
\text { безопасность } \\
0>S_{\rho}^{*}>-\infty\end{array}$ \\
\hline & $E$-состояние & $\begin{array}{c}\text { Гибель } \\
\omega_{\Sigma}^{*}=\infty=\omega_{\infty}\end{array}$ & & & $\begin{array}{c}\text { Абсолютная опасность } \\
S_{\rho}^{*}=-\infty=S_{-\infty}\end{array}$ \\
\hline
\end{tabular}


противоположными соотношениями - в зависимости от анализируемой стратегии эволюции:

$$
R(X)=R\left(\frac{S_{\rho}}{\rho}\right) \text {, или } R(Y)=R\left(\frac{\rho}{S_{\rho}}\right) .
$$

Следовательно, (11) оказывается своеобразным направляющим вектором эволющчи, который вполне описывает (определяет) ее развитие. Оказалось, что этапы существования системы (см. таблицу) связаны с фундаментальными золотыми пропорциями (рисунок 3) [24]. Это позволяет думать, что теория эволюции систем по поврежденности тоже относится к фундаментальным результатам. Детальное изложение этой концепции выходит за рамки данной статьи.

Как отмечено выше, люди изучают определенные события, явления, ситуации на Земле применительно к земным условиям, а затем относительно смело распространяют их так далеко,

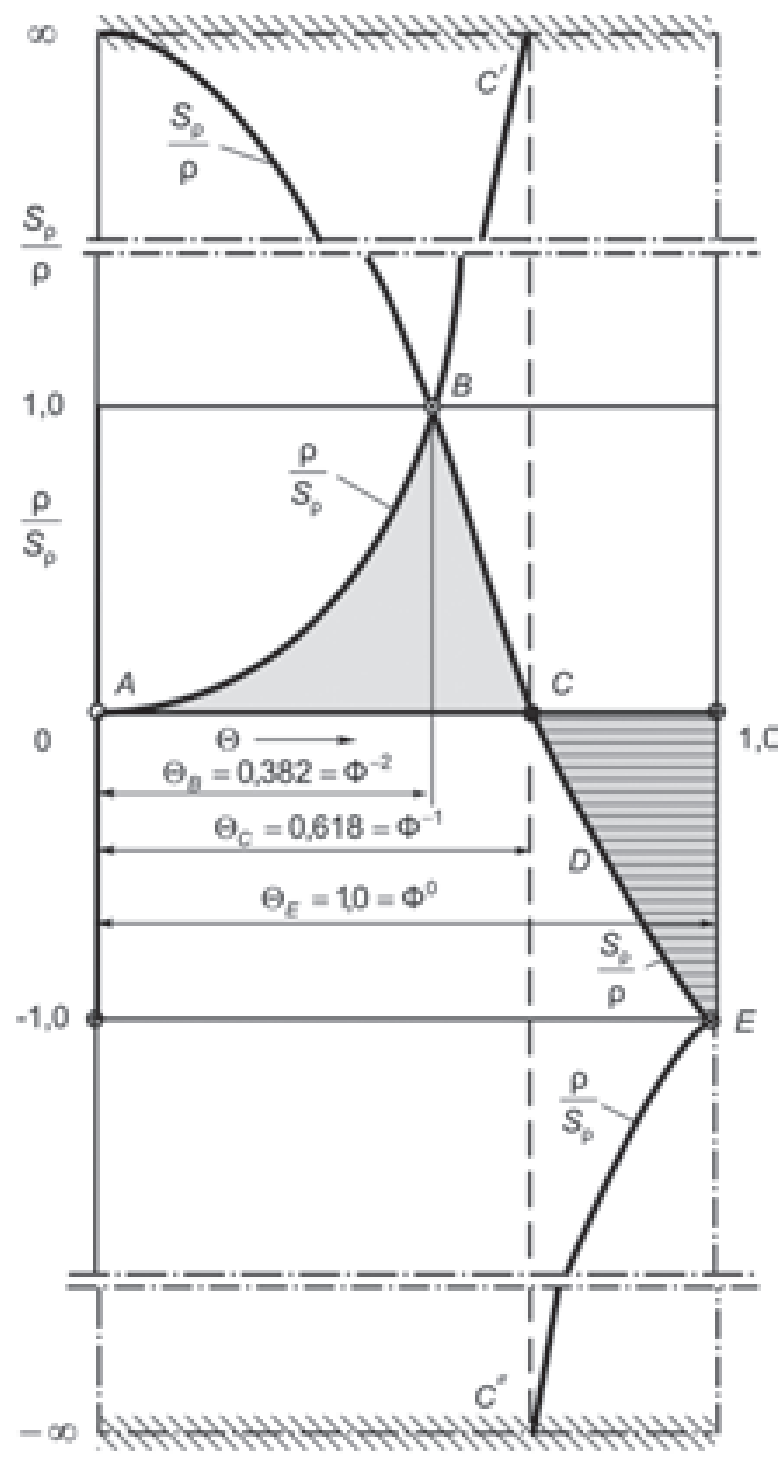

Рисунок 3 - Двуединая стратегия эволюции в относительном временном интервале $0 \leq \theta=t / T_{*} \leq 1$

Figure 3 - A two-pronged evolution strategy in the relative time interval $0 \leq \theta=t / T_{*} \leq 1$ как «можется». Так было, например, с понятием энтропии [7]. Проиллюстрируем сказанное еще одним земным примером - упомянутыми выше прямым и обратнымм эффектами, которые изучают в трибофатике и механотермодинамике $[10,14,15,22]$. Начнем с «земных примеров»: влияние процессов изнашивания на сопротивление усталости (прямой эффект) и противоположное влияние процессов усталостного повреждения на износостойкость (обратный эффект) изучаются в любой трибофатической системе как разные взаимодействия и поэтому различаются принципиально (так, они не могут быть равными или уравновешенными) [13-17]. Механотермодинамическая система [10, 11]: влияние коррозионной среды (или температуры) на изменение предела выносливости углеродистой стали (прямой эффект) никак не тождественно влиянию механических напряжений того же уровня на изменение состояния и свойств среды [25]. Совершенно естественно, что эволюция состояний указанных систем, характеризуемая, например, их ресурсом (долговечностью, параметрами надежности, характером повреждения или разрушения), во всех четырех примерах анализа взаимодействий не уравновешены и различаются кардинально. Но подобные (аналогичные) закономерности (явления) обнаруживаются и во Вселенной, как было описано выше.

Математические модели взаимодействий во Вселенной, по-видимому, можно и нужно строить как диалектические $\Lambda$-взаимодействия (как в трибофатике и механотермодинамике) на макроуровне и/или параметрами неаддитивности (как в q-исчислении) на наноуровне [7]. Естественно, могут быть найдены и другие модели взаимодействия на космическом уровне.

К теории безграничного сжатия. Кратко обсудим и еще одну проблему. Исследования Опnенгеймера и Снайдерса [26] привели к следующему общему выводу. «Звезды больших масс, исчерпавшие свои ядерные источники энергии, коллапсируют под действием собственного гравитационного поля. Если масса звезды не снизится до величины порядка массы Солнца (до предела Чандрасекара: $1,4 \mathrm{M}=$ - прим. авторов), то ее сжатие будет происходить безгранично» - в случае, когда можно пренебречь давлением. Такое сжатие ведет, вероятно, к образованию, в конечном счете, ограниченной среды (или даже точки?) с огромной плотностью материи, т. е. черной дыры.

Однако вывод о безграничном сжатии сделан без учета противодействующего давления - внутреннего сопротивления любого материального объекта. А оно, по-видимому, не может не существовать.

В первом приближении условно будем считать, что это сопротивление описывается законом Гука:

$$
F_{L}=E_{L} \cdot \Delta x
$$


Конкретизируем величину абсолютного сжатия $\Delta x$. Будем считать, что сжимаемая силой $F_{L}$ среда имеет размеры порядка планковской длины $L_{P}$, т. е.

$$
x=\alpha L_{P}, \alpha<<1 .
$$

Если $\alpha^{1} L_{P}$ - начальное, а $\alpha_{2} L_{P}$ - конечное расстояние между точками 1 и 2 , с массами $m_{1}$ и $m_{2}$ среды, получаем две характеристики: абсолютная величина сжатия

$$
\Delta \alpha L_{P}=\alpha_{2} L_{P}-\alpha_{1} L_{P}=\left(\alpha_{2}-\alpha_{1}\right) L_{P}
$$

и относительное сжатие

$$
\varepsilon=\frac{\Delta \alpha L_{p}}{\alpha_{1} L_{p}}=\frac{\alpha_{2}-\alpha_{1}}{\alpha_{1}}=\frac{\alpha_{2}}{\alpha_{1}}-1 .
$$

И тогда закон Гука (13) можно записать, используя для характеристики сопротивления сжатию либо силу $F_{L}$ :

либо давление $p$ :

$$
F_{L}=E_{L}\left(\alpha_{2}-\alpha_{1}\right) L_{P}, \alpha_{2}<\alpha_{1},
$$

$$
p=K_{V L}\left(\frac{\alpha_{2}}{\alpha_{1}}-1\right),
$$

где $K_{V L}$ - модуль объемного сжатия.

Для интервала $0 \leq \alpha_{2} \leq 1$ в критическом случае $\alpha_{2}=0$ прогнозируется:

$$
p_{C}=K_{V L}(-1)=-K_{V L},
$$

т. е. конечное давление равно модулю объемного сжатия с противоположным знаком.

И далее малейший рост сжатия $p>p_{C}$ ведет к своеобразному взрывному распаду («разрушению») среды - скачок, наблюдаемый и на рисунке 3. Что означает (физически) распад среды, сжатие которой превысит ее внутреннее сопротивление сближению («предельное отталкивание») - это вопрос, подлежащий исследованию.

Анализ полученных решений будет дан в сообщении 3. А пока заметим, что критическому значению давления (19) должно соответствовать и критическое значение энтропии, поэтому ожидается и взрывной скачок ее величины при достижении критической плотности среды.

Заключение. Изложена космологическая концепция об аналогии термодинамики и механики черных дыр. Поскольку анализ показал ее недостаточность, дано развитие этой концепции. Система «черная дыра - горизонт событий» представляется как механотермодинамическая неаддитивная мультисистема; изложены подходы и методы оценки ее энтропии.

Авторы данной статьи осознают спорность изложенной гипотезы, но они надеются на ее полезность. Вспомним: Резерфорд опубликовал в свое время спорную (заведомо неверную) гипотезу о строении атома. И она стала стимулом и отправной точкой для новых исследований, результаты которых воплотились в практику современной жизни. Авторы ожидают, что и изложенная гипотеза может быть интересной для новых и перспективных исследований в области космологии.

Выводы по сообщениям 1 и 2. 1. Выполнена систематизация и дан анализ основных (классических) представлений об энтропии, на основе которого изложены современные подходы к ее оценке.

2. Показано, что фундаментальное понятие об энтропии как характеристике рассеяния энергии в среде стало эффективным параметром для анализа эволюции систем не только в термодинамике, но и в ряде смежных научных дисциплин: механике, информатике, трибофатике, механотермодинамике (см. таблицу 1 в [7]).

3. Изложена концепция об аналогии термодинамической энтропии и энтропии черных дыр в космологии. Установлено, что эта концепция требует развития, так как для черной дыры характерно не рассеяние, а поглощение энергии. Предложено использовать для анализа энтропии черных дыр известные представления о трибофатической и механотермодинамической энтропии [7], которые характеризуют поглощение энергии в средах.

4. Дано развитие понятия об энтропии в космологии на примере анализа механотермодинамической системы «горизонт событий - черная дыра». Оценка энтропии горизонта событий дается посредством термодинамической энтропии, а собственно черной дыры - посредством трибофатической энтропии. Энтропия системы «горизонт событий - черная дыра» оценивается как результат $\Lambda$-взаимодействий энтропии компонент системы.

5. Изложенные представления имеют большую практическую значимость. Так, если ранее в трибологии процессы изнашивания и усталостного повреждения и разрушения при механической усталости изучали только опосредованно - с помощью термодинамической энтропии как характеристики рассеяния энергии, то теперь эти процессы изучают непосредственно - с помощью трибофатической энтропии как характеристики поглощения энергии, которая инициирует и расходуется на зарождение и развитие любых необратимых повреждений [27-29]. Практическую значимость таких методов исследования переоценить трудно.

\section{Список литературы}

1. Bekenstein, J.D. Black Holes and Entropy / J.D. Bekenstein // Physical Review. — 1973. - D7. - Pp. 2333-2346.

2. Bekenstein, J.D. Black Holes and Information Theory / J.D. Bekenstein // Contemporary Physics. - 2004. — Vol. 45, iss. 1. - Pp. 31-43.

3. Hawking, S.W. Particle Creation by Black Holes / S.W. Hawking // Communications and Mathematical Physics. - 1975. Vol. 43. - Pp. 199-220.

4. Bardeen, J.M. The Four Laws of Black Hole Mechanics / J.M. Bardeen, B. Carter, S.W. Hawking // Communications and Mathematical Physics. - 1973. - Vol. 31. - Pp. 161-170.

5. Kondepudi, D. Modern Thermodynamics (From Heat Engines to Dissipative Structures) / D. Kondepudi, I. Prigogine. - John Wiley \& Sons, 1998. — 486 p. 
6. Planck, M. Treatise on Thermodynamics / M. Planck. - 3ed. Dover, 1945. - $320 \mathrm{p}$

7. Сосновский, Л.А. Развитие понятия об энтропии: от термодинамики до космологии. Сообщение 1. Понятие об энтропии: термодинамика, механика, информатика, трибофатика, механотермодинамика / Л.А. Сосновский, С.С. Щербаков // Механика машин, механизмов и материалов. - 2020. № 3(52). - C. 78-88.

8. Сосновский, Л.А. Об одном виде энтропии как мере поглощения энергии, расходуемой на производство повреждений в механотермодинамической системе / Л.А. Сосновский // Докл. НАН Беларуси. — 2007. — Т. 51, № 6. - С. 100-104.

9. Сосновский, Л.А. Принципы механотермодинамики: монография / Л.А. Сосновский, С.С. Щербаков. - Гомель: БелГУТ, 2013. - $150 \mathrm{c}$.

10. Sosnovskiy, L.A. Mechanothermodynamics / L.A. Sosnovskiy, S.S. Sherbakov. - Springer, 2016. - $155 \mathrm{p}$.

11. Зельдович, Я.Б. Строение и эволюция Вселенной Я.Б. Зельдович, И.Д. Новиков. - М.: Наука, 1975. — 736 с.

12. Новиков, И.Д. Эволюция Вселенной / И.Д. Новиков. - М.: Наука, 1979. - $176 \mathrm{c}$

13. Сосновский, Л.А. Механика износоусталостного повреждения / Л.А. Сосновский. - Гомель: БелГУТ, 2007. — 434 с.

14. Щербаков, С.С. Механика трибофатических систем С.С. Щербаков, Л.А. Сосновский. - Минск: БГУ, 2011. $407 \mathrm{c}$.

15. Сосновский, Л.А. Фундаментальные и прикладные задачи трибофатики: курс лекций / Л.А. Сосновский, М.А. Журавков, С.С. Щербаков. - Минск: БГУ, 2011. — 488 с.

16. Sosnovskiy, L.A. Tribo-Fatigue. Wear-Fatigue Damage and Its Prediction / L.A. Sosnovskiy // Series: Foundations of Engineering Mechanics. - Springer, 2005. - $424 \mathrm{p}$.

17. Sosnovskiy, L.A. Tribo-Fatigue. Wear-fatigue damage and its prediction (in chinese) / L.A. Sosnovskiy. - Beijing: China University of Mining and Technology Press, 2013. - $324 \mathrm{p}$.

18. О методологии феноменоанализа в механике / М.А. Журавков [и др.] // Теоретич. и прикл. механика: межвед. сб. науч.-метод. ст. / БНТУ. - Минск, 2016. - Вып. 31. - С. 3-10.

19. Сосновский, Л.А. Некоторые соображения о триаде вера знание - мировоззрение / Л.А. Сосновский // Религия и об- разование в светских обществах: материалы междунар. науч. конф., Минск, 27-28 мая 2014 г. - Минск: Право и экономика, 2014. - С. 49-53.

20. Фейнман, Р. Лекции по физике / Р. Фейнман. - М.: Мир, 1963. - $261 \mathrm{c}$.

21. Сосновский, Л.А. Основы теории эволюции неорганических и органических систем, в том числе живых и разумных / Л.А. Сосновский, С.С. Щербаков, А.А. Лазаревич // Национальная философия в глобальном мире: материалы Первого белорус. философского конгр., Минск, 18-20 окт. 2017 г. - Минск: Беларус. навука, 2018. - С. 155-178.

22. Сосновский, Л.А. Механотермодинамика (об объединении великих конкурентов: 1850-2015) / Л.А. Сосновский // Механика машин, механизмов и материалов. - 2016. № 4(37). - C. 19-41.

23. Щербаков, С.С. Модели состояний трибофатических и механотермодинамических систем / С.С. Щербаков // Актуальные вопросы машиноведения: сб. науч. тр. / Объедин. ин-Т машиностроения НАН Беларуси; редкол.: С.Н. Поддубко [и др.]. — 2019. - Вып. 8. - С. 204-208.

24. Сосновский, Л.А. $L$-риск (механотермодинамика необратимых повреждений) / Л.А. Сосновский. - Гомель: БелГУТ, 2004. - $317 \mathrm{c}$.

25. Гутман, Э.М. Механохимия металлов и защита от коррозии / Э.М. Гутман. - М.: Металлургия, 1974. — 230 с.

26. Oppenheimer, J.R. On Continued Gravitational Contraction / J.R. Oppenheimer, H. Snyder // Physical Review. — 1939. Vol. 56. - Pp. 455-459.

27. Sosnovskiy, L.A. A Model of Mechanothetmodynamic Entropy in Tribology / L.A. Sosnovskiy, S.S. Sherbakov // Entropy. 2017. — Vol. 19(3). DOI: https://doi.org/10.3390/e19030115.

28. Sosnovskiy, L.A. Mechanothermodynamic Entropy and Analysis of Damage State of Complex Systems / L.A. Sosnovskiy, S.S. Sherbakov // Entropy. — 2016. — Vol. 18(7). DOI: https:// doi.org/10.3390/e18070268.

29. Sosnovskiy, L.A. On the Development of Mechanothermodynamics as a New Branch of Physics / L.A. Sosnovskiy, S.S. Sherbakov // Entropy. - 2019. - Vol. 21(12). DOI: https://doi.org/10.3390/e21121188.

SOSNOVSKIY Leonid A., D. Sc. in Eng., Prof.

Professor of the Department "Locomotives"

E-mail: tribo-fatigue@mail.ru

SHERBAKOV Sergei S., D. Sc. in Phys. and Math., Prof.

Professor of the Department of Theoretical and Applied Mechanics ${ }^{2}$

E-mail: sherbakovss@mail.ru

${ }^{1}$ Belarusian State University of Transport, Gomel, Republic of Belarus

${ }^{2}$ Belarusian State University, Minsk, Republic of Belarus

Received 25 August 2020.

\section{DEVELOPMENT OF THE CONCEPT OF ENTROPY: FROM THERMODYNAMICS TO COSMOLOGY. PART 2. HYPOTHESIS OF ENTROPY IN COSMOLOGY AND ITS DEVELOPMENT}

In the work [7], the classical concepts of thermodynamic entropy are systematized and modern approaches to assessing the tribo-fatigue and mechanothermodynamic entropy of non-additive systems are outlined. In this article, the concept of the analogy of thermodynamics and mechanics of black holes is presented and analyzed, which made it possible to estimate their (thermodynamic) entropy. The insufficiency of this concept is that thermodynamic entropy is a characteristic of energy dissipation, whereas black holes are characterized by the absorption of energy and matter. In this regard, it is proposed to consider the event horizon as a thermodynamic medium, and a black hole as a tribo-fatigue object. And then the "black hole - event horizon" system is presented as a combined mechanothermodynamic non-additive multisystem. Methods for estimating the total (mechanothermodynamic) entropy and its components - tribo-fatigue and thermodynamic entropy in black hole mechanics are presented. With regard to individual (specific) zones and objects of the universe, the well- 
known theory of Zeldovich is accordingly modified: the universe is a thermodynamic medium with discretely distributed (scattered) dense and/or solid bodies (objects) — stars, galaxies, etc. Behavior of such a system (direct and back effects in the universe) are described. The peculiarity of the action of the medium on the stars and, conversely, the action of the cluster of stars on the interaction between them consists in the fact that it is non-Newtonian: action is not equal to reaction. It is the inequality of action against counteraction, which has radically different mechanisms and consequences (results), or, in other words, the imbalance of the universe that determine its general motion in space-time. The changing set of all states is the evolution of the universe. The analysis of possible strategies for the evolution of mechanothermodynamic systems is carried out on the basis of the fundamental principle: the damageability of everything that exists has no conceivable boundaries. This principle is formulated in mechanothermodynamics and used in philosophy to create a generalized theory of the evolution of the material world.

Keywords: black hole; event horizon; thermodynamics; mechanics of black holes; thermodynamic, tribofatigue, mechanothermodynamic entropy; Bekenstein-Hawking concept; Planck length, area, volume; additive, non-additive system; Zeldovich theory; system evolution by damage; L-risk; $S_{\rho}$-safety; universe

DOI: https://doi.org/10.46864/1995-0470-2020-1-54-80-88

\section{References}

1. Bekenstein J.D. Black holes and entropy. Physical review, 1973, vol. 7, iss. 8, pp. 2333-2346.

2. Bekenstein J.D. Black holes and information theory. Contemporary physics, 2004, vol. 45, iss. 1, pp. 31-43.

3. Hawking S.W. Particle creation by black holes. Communications and mathematical physics, 1975, vol. 43, pp. 199-220.

4. Bardeen J.M., Carter B., Hawking S.W. The four laws of black hole mechanics. Communications and mathematical physics, 1973, vol. 31, pp. 161-170.

5. Kondepudi D., Prigogine I. Modern thermodynamics (from heat engines to dissipative structures). John Wiley \& Sons, 1998. $486 \mathrm{p}$.

6. Planck M. Treatise on thermodynamics. Dover, 1945. 320 p.

7. Sosnovskiy L.A., Sherbakov S.S. Razvitie ponyatiya ob entropii: ot termodinamiki do kosmologii. Soobshchenie 1. Ponyatie ob entropii: termodinamika, mekhanika, informatika, tribofatika, mekhanotermodinamika [Development of the concept of entropy: from thermodynamics to cosmology. Part 1 . The concept of entropy: thermodynamics, mechanics, informatics, tribo-fatigue, mechanothermodynamics]. Mechanics of machines, mechanisms and materials, 2020, no. 3(52), pp. 78-88 (in Russ.).

8. Sosnovskiy L. A. Ob odnom vide entropii kak mere pogloshcheniya energii, raskhoduemoy na proizvodstvo povrezhdeniy $\mathrm{v}$ mekhanotermodinamicheskoy sisteme [On one type of entropy as a measure of energy absorption spent on damage production in a mechanothermodynamic system]. Doklady of the National Academy of Sciences of Belarus, 2007, vol. 51, no. 6, pp. 100-104 (in Russ.).

9. Sosnovskiy L.A., Sherbakov S.S. Printsipy mekhanotermodinamiki [Principles of mechanothermodynamics]. Gomel, Belorusskiy gosudarstvennyy universitet transporta Publ., 2013. 150 p. (in Russ.)

10. Sosnovskiy L.A., Sherbakov S.S. Mechanothermodynamics. Springer, 2016. 155 p.

11. Zeldovich Ya.B., Novikov I.D. Stroenie i evolyutsiya Vselennoy [Structure and evolution of the universe]. Moscow, Nauka Publ., 1975. 736 p. (in Russ.).

12. Novikov I.D. Evolyutsiya Vselennoy [Evolution of the universe]. Moscow, Nauka Publ., 1979. 176 p. (in Russ.).

13. Sosnovskiy L.A. Mekhanika iznosoustalostnogo povrezhdeniya [Mechanics of wear-fatigue damage]. Gomel, Belorusskiy gosudarstvennyy universitet transporta Publ., 2007. 434 p. (in Russ.).

14. Sherbakov S.S., Sosnovskiy L.A. Mekhanika tribofaticheskikh sistem [Mechanics of tribo-fatigue systems]. Minsk, Belorusskiy gosudarstvennyy universitet Publ., 2011. 407 p. (in Russ.).

15. Sosnovskiy L.A., Zhuravkov M.A., Sherbakov S.S. Fundamentalnye $i$ prikladnye zadachi tribofatiki [Fundamental and applied problems of tribo-fatigue]. Minsk, Belorusskiy gosudarstvennyy universitet Publ., 2011. 488 p. (in Russ.).

16. Sosnovskiy L.A. Tribo-fatigue. Wear-fatigue damage and its prediction. Springer, 2005. 424 p.
17. Sosnovskiy L.A. Tribo-Fatigue. Wear-fatigue damage and its prediction (in chinese). Beijing, China University of Mining and Technology Press, 2013. 324 p.

18. Zhuravkov M.A., Sherbakov S.S., Makhutov N.A., Sosnovskiy L.A. O metodologii fenomenoanaliza v mekhanike [On the methodology of phenomenoanalysis in mechanics]. Teoreticheskaya i prikladnaya mekhanika, 2016, iss. 31, pp. 3-10 (in Russ.).

19. Sosnovskiy L.A. Nekotorye soobrazheniya o triade vera - znaniemirovozzrenie [Some considerations about the triad faith knowledge - worldview]. Materialy Mezhdunarodnoy nauchnoy konferentsii "Religiya i obrazovanie v svetskikh obshchestvakh", [Proc. International scientific conference "Religion and education in secular societies"]. Minsk, 2014, pp. 49-53 (in Russ.).

20. Feynman R. Lektsii po fizike. Tom 4 [Lectures in physics. Volume 4]. Moscow, Mir Publ., 1963. 261 p. (in Russ.).

21. Sosnovskiy L.A., Sherbakov S.S., Lazarevich A.A. Osnovy teorii evolyutsii neorganicheskikh i organicheskikh sistem, v tom chisle zhivykh i razumnykh [Fundamentals of the theory of evolution of inorganic and organic systems, including living and intelligent]. Materialy pervogo belorusskogo filosofskogo kongressa "Natsionalnaya filosofiya v globalnom mire" [Proc. 1st Belarusian philosophical congress "National philosophy in the global world"']. Minsk, 2017, pp. 155-178 (in Russ.).

22. Sosnovskiy L.A. Mekhanotermodinamika (ob obedinenii velikikh konkurentov: 1850-2015) [Mechanothermodynamics (about the unification of great competitors: 1850-2015)]. Mechanics of machines, mechanisms and materials, 2016, no. 4(37), pp. 19-41 (in Russ.).

23. Sherbakov S.S. Modeli sostoyaniy tribofaticheskikh i mekhanotermodinamicheskikh sistem [Models of states of tribo-fatigue and mechanothermodynamic systems]. Aktualnye voprosy mashinostroeniya, 2019, iss. 8, pp. 204-208 (in Russ.).

24. Sosnovskiy L.A. L-Risk (mekhanotermodinamika neobratimykh povrezhdeniy) [L-risk (mechanothermodynamics of irreversible damage)]. Gomel, Belorusskiy gosudarstvennyy universitet transporta Publ., 2004. 317 p. (in Russ.).

25. Gutman E.M. Mekhanokhimiya metallov $i$ zashchita ot korrozii [Mechanochemistry of metals and protection against corrosion]. Moscow, Metallurgiya Publ., 1974. 230 p. (in Russ.).

26. Oppenheimer J.R., Snyder H. On continued gravitational contraction. Physical review, 1939, vol. 56, pp. 455-459.

27. Sosnovskiy L.A., Sherbakov S.S. A model of mechanothermodynamic entropy in tribology. Entropy, 2017, vol. 19(3). DOI: https://doi.org/10.3390/e19030115.

28. Sosnovskiy L.A., Sherbakov S.S. Mechanothermodynamic entropy and analysis of damage state of complex systems. Entropy, 2016, vol. 18(7). DOI: https://doi.org/10.3390/e18070268.

29. Sosnovskiy L.A., Sherbakov S.S. On the development of mechanothermodynamics as a new branch of physics. Entropy, 2019, vol. 21(12). DOI: https://doi.org/10.3390/e21121188. 